Research

Open Access

\title{
A transcriptome map of cellular transformation by the fos oncogene Jared M Ordway ${ }^{\dagger 2}$, Steven D Fenster ${ }^{\dagger 1}$, Hong Ruan ${ }^{1}$ and Thomas Curran*1
} Address: ${ }^{1}$ Department of Developmental Neurobiology, St. Jude Children's Research Hospital, 332 N. Lauderdale St., Memphis, TN, 38105, USA
and ${ }^{2}$ Orion Genomics, 4041 Forest Park Ave., St. Louis, MO, 63108, USA

Email: Jared M Ordway - jordway@oriongenomics.com; Steven D Fenster - steven.fenster@stjude.org; Hong Ruan - hong.ruan@stjude.org; Thomas Curran* - thomas.curran@stjude.org

* Corresponding author †Equal contributors

Published: 26 May 2005

Molecular Cancer 2005, 4:19 doi:10.1186/1476-4598-4-19
Received: 0I April 2005

Accepted: 26 May 2005

This article is available from: http://www.molecular-cancer.com/content/4/1/19

(C) 2005 Ordway et al; licensee BioMed Central Ltd.

This is an Open Access article distributed under the terms of the Creative Commons Attribution License (http://creativecommons.org/licenses/by/2.0), which permits unrestricted use, distribution, and reproduction in any medium, provided the original work is properly cited.

\begin{abstract}
Background: The c-fos gene was originally identified as the cellular homolog of the oncogene $v$ fos carried by the Finkel-Biskis-Jenkins and Finkel-Biskis-Reilly murine osteogenic sarcoma retroviruses. Sustained expression of fos is sufficient to induce cellular transformation in vitro and tumorigenesis in vivo. Fos functions as a component of the AP-I transcription factor complex to regulate gene transcription and several differentially expressed genes have been identified in cells transformed by fos. We have extended these studies by constructing a cellular system for conditional transformation by v-fos. Using Affymetrix-based DNA microarray technology, we analyzed transcriptional changes over the course of transformation and reversion in an inducible $\mathrm{v}$ fos system.
\end{abstract}

Results: Microarray analyses of temporal gene expression during the process of $v$-fos mediated cellular transformation and morphological reversion revealed a remarkably dynamic transcriptome. Of the more than 8000 genes analyzed in this study, 3766 genes were categorized into 18 geneexpression patterns by using self-organizing map analysis. By combining the analysis of gene expression profiles in stably transformed cells with the analysis of sequential expression patterns during conditional transformation, we identified a relatively small cohort of genes implicated in $v$ fos mediated cellular transformation.

Conclusion: This approach defines a general conditional cell transformation system that can be used to study the endogenous transcription regulatory mechanisms involved in transformation and tumorigenesis. In addition, this study is the first reported analysis of dynamic changes in gene expression throughout experimentally controlled morphological transformation mediated by $\mathrm{v}$-fos.

\section{Background}

The c-fos proto-oncogene encodes an immediate-early transcription factor that is rapidly and transiently induced by a variety of extracellular stimuli associated with cellular responses such as proliferation, differentiation, apoptosis and neuronal signalling [1]. The c-Fos protein functions by forming leucine zipper dimers with members of the Jun and ATF/CREB families that comprise the transcription factor complexes collectively referred to as AP-1 [2]. The tightly regulated expression and activity of AP- 1 family members defines a prototypical mechanism whereby short-term extracellular signals are coupled to appropriate 
long-term changes in cellular phenotype by selective regulation of gene expression.

The identification of $\mathrm{v}$-fos as the oncogene carried by the Finkel-Biskis-Jenkins and Finkel-Biskis-Reilly murine osteosarcoma retroviruses contributed to the realization that tumorigenic retroviruses harbor viral versions of cellular genes and that these genes can elude the regulatory constraints imposed upon the endogenous gene [3-5]. The viral fos oncogenes contain point mutations and deletions that enhance their transforming potential [6]. However, sustained expression of c-fos is sufficient to induce cellular transformation in vitro and tumorigenesis in vivo [7]. Therefore, fos-induced transformation and tumorigenesis is the consequence of inappropriate fos activity within susceptible cells rather than a gain-of-function mechanism specific to the viral fos oncogene.

Many signal transduction pathways implicated in tumorigenesis functionally converge on activation of $\mathrm{c}-\mathrm{fos}$ and $\mathrm{AP}-1$, suggesting that inappropriate activation of $\mathrm{c}-$ fos contributes to various aspects of tumorigenesis. This contribution involves direct transcriptional regulation of AP-1 target genes and secondary mechanisms of transcriptional regulation. For example, increased expression and activity of Dnmt1, a DNA methyltransferase that methylates CpG dinucleotides [8], is necessary for morphological transformation by c-fos [9]. CpG methylation within promoter regions functions as an epigenetic mark that establishes or maintains transcriptional repression by recruiting chromatin modification machinery [10]. A previous study identified specific genes that are irreversibly silenced in association with DNA hypermethylation in fos-transformed cells [11]. Therefore, during fos-mediated transformation, there is conditional deregulation of target gene expression dependent upon continual oncogene activity, in addition to long-term epigenetic reprogramming of gene expression that can persist even when the direct effects of oncogene activity are suppressed.

Studies of stably transformed cell lines have found gene expression changes associated with fos transformation and have yielded functional data that implicate differentially expressed genes in aspects of oncogenic transformation [12-14]. In the study described here, we took advantage of a conditional cellular system (Laclv-fos) that allows control of $\mathrm{v}$-fos expression and morphologic transformation. This approach refines the analysis of gene expression associated with fos transformation by distinguishing gene expression changes coincident with morphological transformation from those that are potentially associated with clonal variation or phenotypic changes that occur downstream of the transformation process. Comparisons of temporal gene expression patterns during conditional cellular transformation with transcriptome profiles of cells stably transformed by c-fos and v-fos revealed a cohort of genes likely to be critical for induction and maintenance of cellular transformation.

\section{Results \\ Inducible laclv-fos system}

In the LacIv-fos cell system, the control of FBJ/R v-fos expression is dependent on the presence of isopropyl-bD-thiogalactopryanoside (IPTG) in the cell culture medium [11]. In the presence of $5 \mathrm{mM}$ IPTG, LacIv-fos cells did not express v-Fos protein detectable by Western blot analysis (Figure 1a). When IPTG was washed away, LacIv-fos cells expressed v-Fos protein with peak expression detected at 72 hours following removal of IPTG and progressive loss of $\mathrm{v}$-Fos protein levels upon re-addition of IPTG. In addition, cells were morphologically transformed within a 72-hour period. Transformation was indicated by an overall change in cell shape that led to a more rounded and light refractory morphology as well as dramatic cytoskeletal alterations (Figure 1b). When IPTG was added back to these transformed cells, v-Fos expression was again repressed and the cells returned to their original morphology within a 72-hour reversion period.

\section{Self-organizing map analysis}

The ability to control both v-Fos expression and morphological transformation in vitro provides an opportunity to investigate global gene expression changes relative to the temporal v-Fos cellular transformation and reversion process. RNA was extracted from LacIv-fos cells treated with $5 \mathrm{mM}$ IPTG at time zero $(\mathrm{t}=0)$; from cells at 24,48 and 72 hours following the removal of IPTG (transforming); and from cells at 24, 48 and 72 hours after the readdition of $5 \mathrm{mM}$ IPTG (reverting). Previous studies have demonstrated that the addition and removal of IPTG does not itself induce persistent changes in gene expression in the parental 208F rat fibroblast cell line $[9,11]$. RNA samples were processed and analyzed by hybridization to Affymetrix rat U34A GeneChip microarrays.

Self-organizing map (SOM) analysis is a powerful tool that can be used to categorize gene expression data into groups that share common temporal expression profiles [15-17]. To identify patterns of gene expression, we created an SOM by using the LacIv-fos microarray data obtained at the seven time points throughout v-Fos transformation and reversion (Figure 2a). The data were mapped into 18 groups that provided the greatest pattern distinction among groups as well as the best pattern consistency within groups. Several assumptions were made on the basis of simple visualization of the map. For example, the expression of genes grouped into pattern 1 peaked 24 hours following removal of IPTG, as well as at 24 hours following re-addition of IPTG. This group likely represents genes whose expression was induced within 24 
A
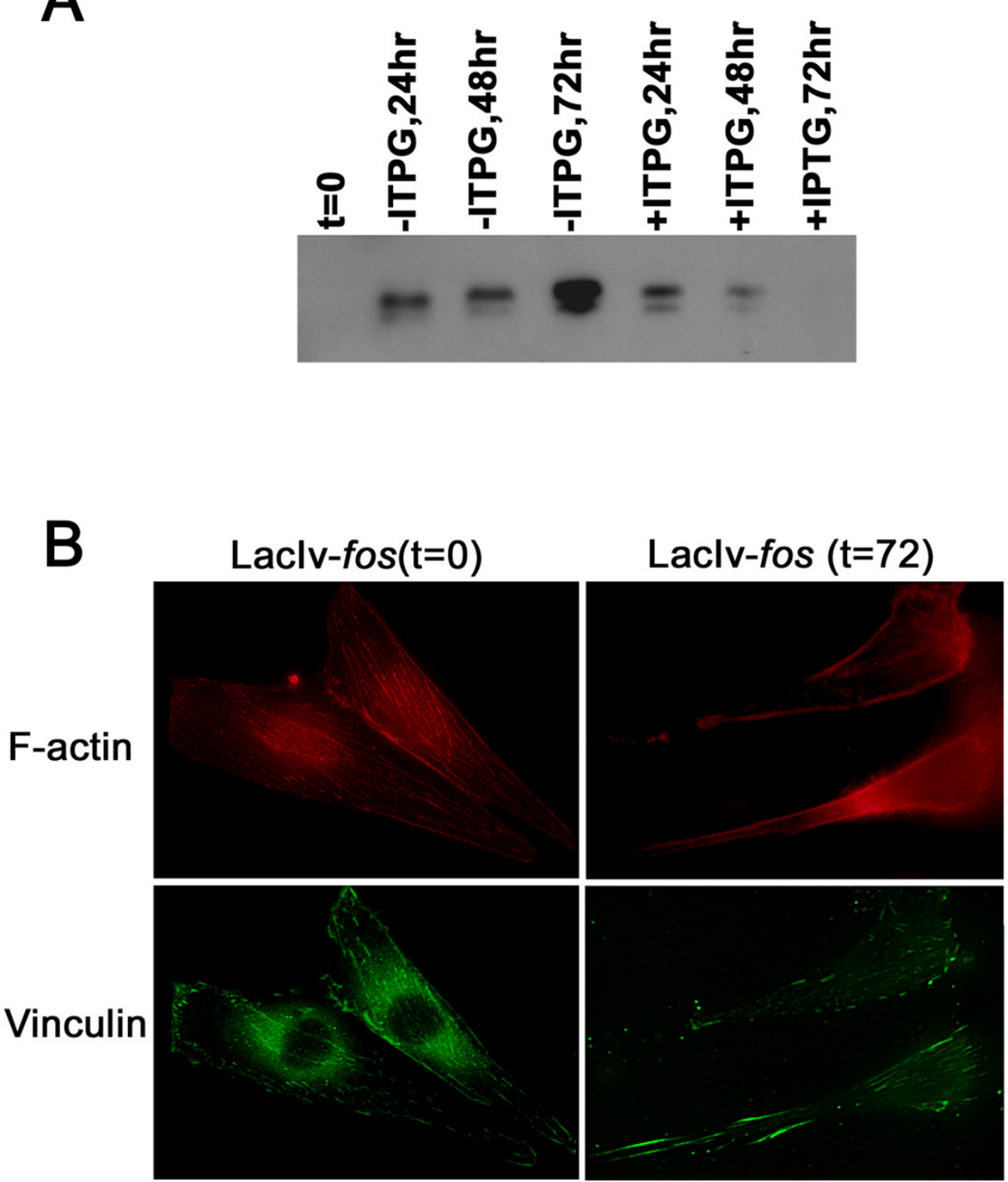

\section{Figure I}

Expression of v-Fos and cell morphology rearrangement in Laclv-fos system during conditional transformation and reversion. (A) Expression of v-Fos protein expression during conditional morphological transformation of Laclv-fos cells. At time zero $(\mathrm{t}=0)$, Fos protein was not detectable in cells. 24 hours after removal of IPTG, Fos protein expression was induced and the immunoreactivity increased over the 72 hour transformation period. After re-addition of IPTG, Fos protein levels rapidly decreased with no detectable signal observed after 72 hours of reversion. (B) Induction of v-Fos expression resulted in dramatic cytoskeletal changes in Laclv-fos cells. Cells were stained with anti-vinculin antibody (green) to detect focal adhesion contact sites and phalloidin (red) was used to track alterations in the actin cytoskeleton. In the presence of IPTG $(\mathrm{t}=$ 0 ), cells have well established focal adhesion sites and coordinated F-actin staining. Seventy-two hours after removal of IPTG ( $t$ $=72$ ), cells lacked defined focal adhesion contacts and displayed disorganized F-actin staining 
A.

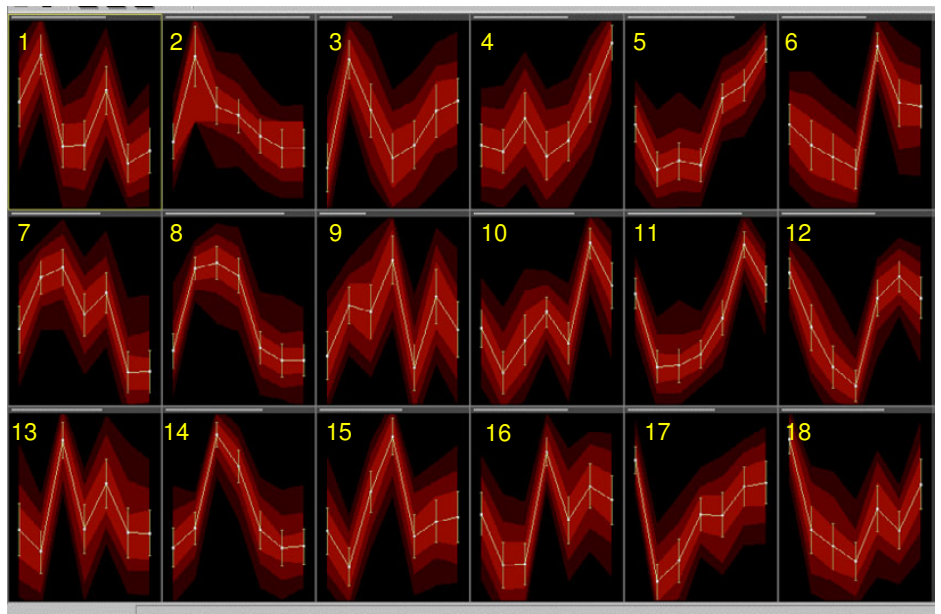

B.

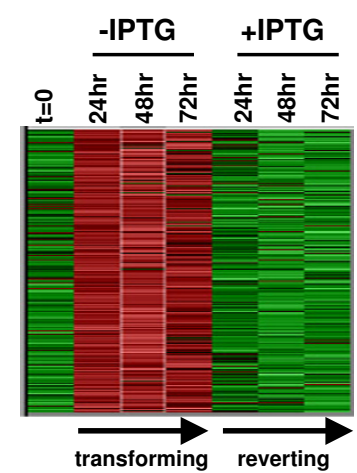

D.

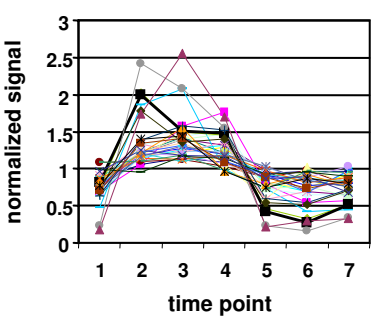

C.
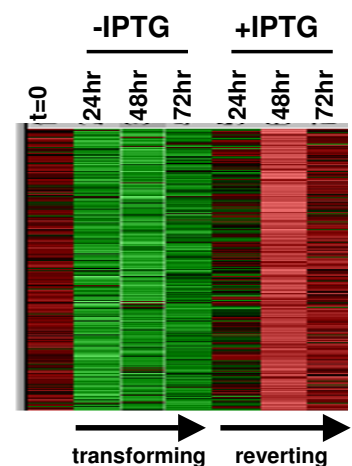

E.

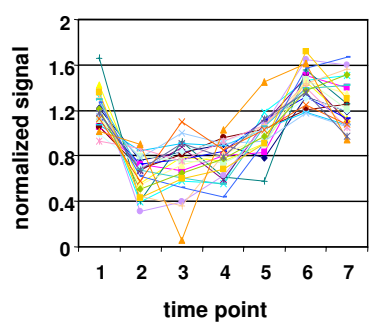

\section{Figure 2}

Self-organizing map (SOM) analysis of microarray expression data in the Laclv-fos system during morphological transformation and reversion. (A) Results of SOM analysis of genes depicting co-regulated clusters of genes across the seven time points throughout v-Fos mediated morphological transformation and reversion. Genes whose expression did not change significantly across time points were eliminated by using a variance filter (see Methods). The 3766 probe sets (out of a possible 8799) that passed the variation filter were grouped into 18 clustered patterns. Blue bars at the top of each graph represent the relative number of probe sets included within the SOM bin. (B) A gene-expression heat map of genes within group 8. This bin represents genes dramatically upregulated specifically during the 72 hour v-Fos transformation period (-IPTG). (C) Gene-expression heat map of genes within group II. This bin represents genes displaying sustained downregulation during the $72 \mathrm{hr} v$-Fos transformation period (+ITPG). Red signal represents upregulated genes and green represents downregulated genes. (D-E) Normalized expression values of all genes within group 8 (D) or group II (E) are plotted to demonstrate the reliability of the representative SOM patterns shown in panel A. 
hours following culture media change rather than genes whose expression coincided with v-Fos-mediated transformation. Indeed, genes represented by pattern 1 included serum-responsive genes such as c-Jun and cyclin D1. However, other patterns included genes that were regulated in a manner more consistent with conditional vFos transformation and reversion. For example, pattern 8 included genes that were dramatically upregulated during the 3-day transformation process, yet return to baseline levels during the three-day reversion process (Figure $2 \mathrm{~b}$ ). This group included two probe sets specific for FBR $\mathrm{v}$-fos included on the Affymetrix U34A GeneChip. These probe sets give a reliable measurement of $\mathrm{FBJ} / \mathrm{R} \mathrm{v}$-fos expression and they did not cross-hybridize with endogenous c-fos transcripts [11]. Conversely, group 11 represents genes that were conditionally downregulated specifically during conditional v-Fos transformation (Figure 2c). Plots of the normalized signal values for each probe set included in these groups further confirmed the reliability of the classifications of gene expression profiles within the SOM (Figure $2 \mathrm{~d}-\mathrm{e})$.

Patterns within the SOM that are consistent with v-Fos transformation and reversion were selected on the basis of the representative expression pattern of each SOM group. For candidate genes whose expression was upregulated, the standard deviation of the mean signal values at a minimum of two of the three time points in the absence of IPTG (transforming) had to be greater than that of the mean signal values at time zero (i.e., the time prior to removal of IPTG from the culture medium). Also, the standard deviation of the mean signal values at a minimum of two of the three final time points in the presence of IPTG (reverting) had to be below that of the mean signal value of the final time point during transformation (i.e., 72 hours after removal of IPTG from the culture medium). For candidate genes whose expression was downregulated, the standard deviation of the mean signal values at a minimum of two of the three transforming time points had to be lower than that of the mean signal values at time zero, and the standard deviation of the mean signal values at a minimum of two of the three time points during reversion had to be higher than that of the final time point during transformation. On the basis of these criteria, patterns 7, 8, 14 and 15 (transforming) and patterns 5, 11, 12, 17 and 18 (reverting) were selected for further analysis. Each of these groups consisted of a large number of genes (Figure 2a). These groups likely included not only genes functionally involved in cellular transformation, but also genes that share similar expression profiles during conditional transformation and reversion yet have no role in the process or maintenance of cellular transformation itself. Interestingly, both patterns 5 and 7 included genes with higher signal-ratio values at the end of morphological reversion ( $t=72$ hours, reverting) than prior to $\mathrm{v}$-fos induction $(\mathrm{t}=0)$. This difference in transcript levels at the beginning of the time course relative to the final time point may be related to a requirement for a higher level of expression of particular genes during the reversion of morphological transformation than during maintenance of the non-transformed state. Alternatively, this may reflect a type of over-compensation in that expression levels within particular expression pattern groups and transcript levels may not completely return to steady-state within the three-day reversion period.

\section{Stable versus inducible $\boldsymbol{v}$-fos expression}

To identify only the most promising candidate genes, we compared these datasets with gene expression profiles of the parental $208 \mathrm{~F}$ cells and $208 \mathrm{~F}$ cells transformed by stable expression of either c-Fos (CMVc-fos cells) or v-Fos (FBJ/R cells). Cells transformed by stable expression of cFos or v-Fos exhibit a large number of differentially expressed genes. For example, when differential expression was defined as a 2-fold increase or decrease based on a comparison with expression in $208 \mathrm{~F}$ cells and when probe sets for expressed sequence tags (ESTs) were excluded, 70 probe sets were scored as upregulated and 104 were scored as downregulated in both CMVc-fos and FBJ/R cells [11]. However, eliminating genes whose expression changes are consistent in both stably and conditionally transformed cell model systems can reduce the level of nonspecific transcriptional variation. Of the 174 differentially expressed probe sets identified in comparisons of both CMVc-fos and FBJ/R cells to $208 \mathrm{~F}$ cells, 63 (36\%) were also categorized into LacIv-fos SOM patterns consistent with increased or decreased expression during conditional v-Fos transformation. Therefore, the majority of gene expression changes associated with stably Fos-transformed cell lines were not recapitulated during conditional v-Fos transformation and reversion. These findings emphasize the value of combining analyses of stable and conditional cellular transformation systems.

To address the reproducibility of gene expression changes during conditional v-Fos transformation, we performed microarray analysis using RNA samples isolated from LacIv-fos cells at time points during a second LacIv-fos transformation and reversion time course experiment. To maintain stringent biological replication of the experiment, these seven time points were treated as an independent experiment rather than as expression values that could be averaged between experiments. Expression patterns of genes differentially expressed in both CMVc-fos and FBJ/R cells relative to $208 \mathrm{~F}$ cells, as well as conditionally regulated within the LacIv-fos SOM obtained from the first experiment, were compared to expression patterns obtained from the independent LacIv-fos transformation and reversion time course experiment. Of the 63 probe sets representing differentially expression in stably Fos- 
A.
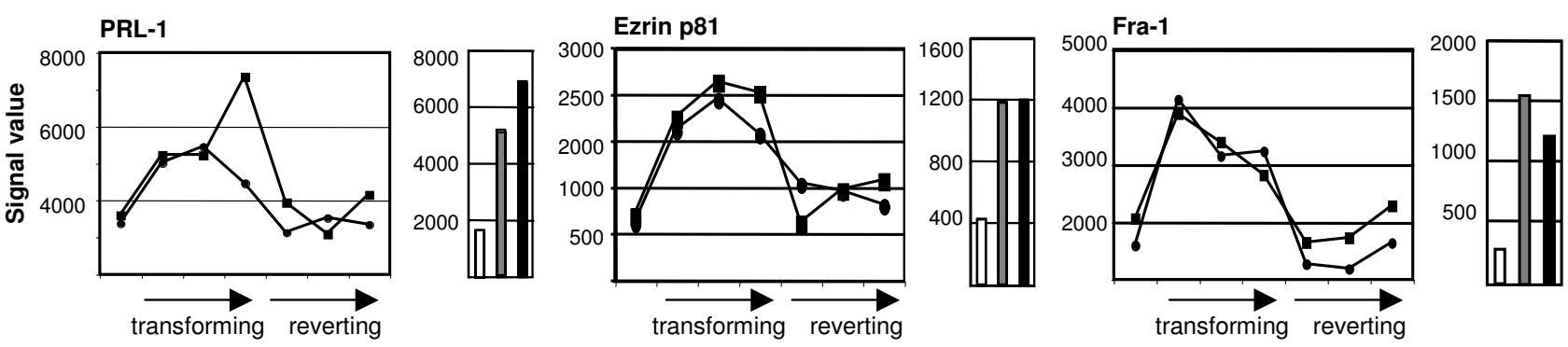

B.
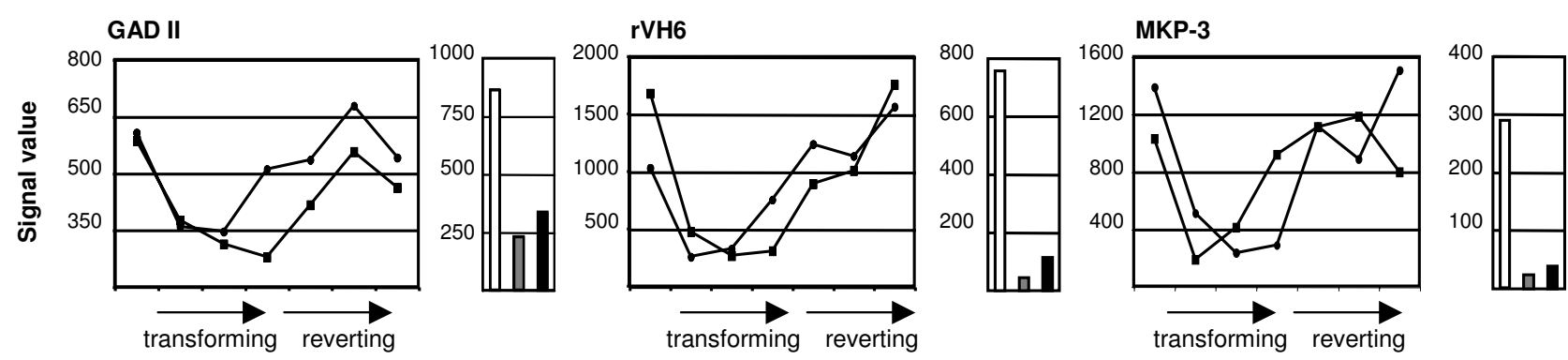

Figure 3

Expression of representative genes conditionally regulated in Laclv-fos cells and differentially expressed in both CMVc-fos cells and FBJ/R cells. Expression patterns of selected genes upregulated (A) or downregulated (B) specifically during conditional transformation, as well as in cells stably transformed by either c-Fos or v-Fos. Gene names are indicated above each pair of graphs. Line graphs indicate raw signal values obtained from the two independent Laclv-fos time course experiments. Time points at 24, 48 and 72 hours in the absence of IPTG (transforming) and at 24, 48 and 72 hours after the re-addition of IPTG (reverting) are indicated by arrows below each line graph. Bar graphs indicate raw signal values obtained in microarray analyses of the parental 208F fibroblast cell line (white), the stably transformed c-fos cell line (CMVc-fos; grey) and the stably transformed v-fos cell line (FBJ/R; black).

transformed cells and conditional regulation in the LacIvfos SOM, 40 (64\%) demonstrated a reproducible temporal profile of gene expression in the independent LacIv-fos transformation and reversion time course experiment (Additional file 1 and Figure 3). In addition, a previous study indicated by Northern blot analysis that representative genes (i.e. TGF- $\beta 3$ and CAIII) had nearly identical patterns of gene expression when compared to microarray analysis [11]. Therefore, the combination of gene expression analysis of stably transformed cells with the analysis of gene expression in an experimentally controlled conditional transformation system is a useful approach to reduce complicating transcriptional variation inherent in any single cell model system.

\section{c-fos versus v-fos gene regulation}

Unlike c-fos, v-fos contains several deletions and point mutations that affect its oncogenic potential [18]. Although sustained expression of c-fos is capable of inducing cellular transformation and tumorigenesis, $\mathrm{v}$-fos is a much more potent oncogene [7]. The increased tumorigenic potential of $\mathrm{v}$-Fos suggests that it directly or indirectly influences the expression of genes not affected by cFos. Consistent with this hypothesis, gene expression 
Table I: Genes whose expression was upregulated (i.e., increased by a factor $\geq 2$ ) in FBJ/R cells (but not in CMVc-fos cells) and was conditionally regulated in Laclv-fos cells.

\begin{tabular}{|c|c|c|}
\hline \multicolumn{3}{|c|}{ Upregulated v-Fos specific candidate genes } \\
\hline GenBank Accession No. & Gene & Pattern \\
\hline AFI00470 & ribosome attached membrane protein 4 (RAMP4) & 7 \\
\hline AF033027 & prenylated SNARE protein Ykt6p (Ykt6) & 7 \\
\hline AF036537 & homocysteine respondent protein HCYP2 & 7 \\
\hline AF054618 & cortactin isoform C & 7 \\
\hline D00092 & mRNA for 70 kd mitochondrial autoantigen & 7 \\
\hline D30740 & 14-3-3 protein mRNA for mitochondrial import stimulation factor (MSF) SI subunit & 7 \\
\hline$M 21476$ & iodothyronine 5-monodeiodinase (5-MD) & 7 \\
\hline M96630 & sec6I homologue & 7 \\
\hline U49930 & ICE-like cysteine protease, Lice (aka Caspase 3) & 7 \\
\hline U95I6I & nuclear protein E3-3 orf2 & 7 \\
\hline$\times 13722$ & LDL-receptor & 7 \\
\hline X7087I & cyclin G & 7 \\
\hline X92097 & transmembrane protein rnp2I.4 & 7 \\
\hline Y09332 & cytosolic peroxisome proliferator-induced acyl-CoA thioesterase & 7 \\
\hline $\mathrm{ABO} 15432$ & LATI (L-type amino acid transporter I & 8 \\
\hline AF069782 & unknown mRNA (aka NAP65) & 8 \\
\hline DI6479 & mitochondrial long-chain 3-ketoacyl-CoA thiolase beta-subunit of mitochondrial trifunctional protein & 8 \\
\hline D26393 & HK2 gene for type II hexokinase* & 8 \\
\hline LI 2025 & tumor-associated glycoprotein E4 (Tage4) & 8 \\
\hline LI 2382 & ADP-ribosylation factor 3 & 8 \\
\hline LI9698 & GTP-binding protein ( $\mathrm{ral}$ A) & 8 \\
\hline L38644 & karyopherin beta & 8 \\
\hline M62992 & glycoprotein p62 & 8 \\
\hline U21718 & clone C426 intestinal epithelium proliferating cell-associated mRNA & 8 \\
\hline U38253 & initiation factor elF-2B gamma subunit (elF-2B gamma) & 8 \\
\hline X82445 & CI5 mRNA & 8 \\
\hline Y00396 & c-myc oncogene & 8 \\
\hline M58587 & interleukin 6 receptor ligand binding chain & 14 \\
\hline M65253 & transformation-associated protein, 34A (aka MMPI0) & 14 \\
\hline UI790I & phospholipase A-2-activating protein (plap) & 14 \\
\hline U35774 & cytosolic branch chain aminotransferase I, cytosolic & 14 \\
\hline U9208I & epithelial cell transmembrane protein antigen precursor (RTI40) & 14 \\
\hline DI2498 & FGF receptor-I* & 15 \\
\hline M74223 & VGF mRNA & 15 \\
\hline S54008 & fibroblast growth factor receptor I beta-isoform* & 15 \\
\hline S56464 & hexokinase II (HK2)* & 15 \\
\hline S81025 & UDP-galactose:N-acetylglucosamine beta-I,4-galactosyltransferase homolog & 15 \\
\hline j05166 & band $3 \mathrm{Cl}-/ \mathrm{HCO} 3$ - exchanger, B3RP2 (aka Slc4a2) & 15 \\
\hline
\end{tabular}

profile analyses to identify genes differentially expressed by a 2-fold margin in FBJ/R cells, but not in CMVc-fos cells, revealed a dramatic increase in the number of transcriptionally upregulated genes in $\mathrm{FBJ} / \mathrm{R}$ relative to $\mathrm{CMVC}$ fos cells: 337 probe sets were scored as specifically upregulated in FBJ/R cells, whereas only 70 probe sets common to CMVc-fos and FBJ/R cells were considered as increased. In contrast, 61 probe sets were scored as downregulated specifically in $\mathrm{FBJ} / \mathrm{R}$ cells but 104 probe sets in both CMVc-fos and FBJ/R cells were considered as downregulated [11]. These FBJ/R cell-specific changes in gene expression were compared with the LacIv-fos SOM and the results of the independent LacIv-fos transformation and reversion time course as described previously. These comparisons revealed an additional 38 upregulated and 29 downregulated probe sets that are differentially regulated in $\mathrm{v}$-fos-transformed cells, but not in c-fos transformed cells (Tables 1 and 2, Figure 4).

\section{Laclv-fos web-accessible database}

The identification of conditionally regulated genes within this system provides potentially unique opportunities to investigate precise transcriptional regulatory mechanisms of endogenous genes within an experimentally amenable cellular context. Therefore, we assembled these datasets into a web-accessible database equipped with various 
Table 2: Genes whose expression was downregulated (i.e., decreased by a factor $\geq 2$ ) in FBJ/R cells (but not in CMVc-fos cells) and was conditionally regulated in Laclv-fos cells.

\begin{tabular}{|c|c|c|}
\hline \multicolumn{3}{|c|}{ Downregulated v-Fos-specific candidate genes } \\
\hline GenBank Accession No. & Gene & Pattern \\
\hline M64780 & agrin & 5 \\
\hline M38I35 & cathepsin $\mathrm{H}$ (RCHII) & 11 \\
\hline U52663 & peptidylglycine alpha-amidating monooxygenase (PAM) & 11 \\
\hline U75917 & clathrin-associated protein 17 (API7) & 11 \\
\hline U75929 & SPARC (aka osteonectin)* & 11 \\
\hline X0534I & 3-oxoacyl-CoA thiolase & 11 \\
\hline DI0026 & glutathione S-transferase Yrs-Yrs & 12 \\
\hline J0279| & acyl coenzyme A dehydrogenase medium chain & 12 \\
\hline j03752 & glutathione S-transferase & 12 \\
\hline M93257 & cathechol-O-methyltransferase & 12 \\
\hline$\times 05472$ & Rat $2.4 \mathrm{~kb}$ repeat DNA right terminal region & 12 \\
\hline X74593 & sorbitol dehydrogenase & 12 \\
\hline Y09333 & mitochondrial very-long-chain acyl-CoA thioesterase & 12 \\
\hline AF034218 & hyaluronidase (Hyal2) & 17 \\
\hline AF065387 & vitamin K-dependent gamma-glutamyl carboxylase & 17 \\
\hline j02810 & prostate glutathione S-transferase & 17 \\
\hline j05031 & isovaleryl-CoA dehydrogenase (IVD) & 17 \\
\hline L01702 & protein-tryosine-phosphatase (LRP) & 17 \\
\hline U10357 & pyruvate dehydrogenase kinase 2 subunit p45 (PDK2) & 17 \\
\hline U2565I & phosphofructokinase muscle isozyme & 17 \\
\hline U75928 & SPARC (aka osteonectin)* & 17 \\
\hline Y $137 \mid 4$ & osteonectin (aka SPARC)* & 17 \\
\hline $\mathrm{D} 00512$ & mitochondrial acetoacetyl-CoA thiolase precursor & 18 \\
\hline DI392I & mitochondrial acetoacetyl-CoA thiolase & 18 \\
\hline D16309 & cyclin D3 & 18 \\
\hline M6092I & NGF-inducible anti-proliferative putative secreted protein (PC3) & 18 \\
\hline S7259 & tissue inhibitor of metalloproteinase type 2 & 18 \\
\hline X95986 & CBR gene & 18 \\
\hline
\end{tabular}

query functions so that users can mine the data to address questions relevant to their own specific interests (see methods). The initial interface of the database is the LacIvfos SOM (Figure 2a). Users can select SOM patterns of interest to obtain a list of all probe sets mapped within that common pattern of gene expression. Each probe set is linked to the raw Affymetrix data for both independent LacIv-fos time course experiments. This arrangement provides an opportunity for convenient confirmation of consistent regulation between the independent time course experiments. In addition, users can query the database for information about specific genes by using Genbank accession numbers or by using genes name as keywords. Keyword queries may include the qualifier functions "and" or "or". These queries will provide a list of all probe sets matching the supplied criteria, and each entry is again linked to the raw Affymetrix data obtained from both independent LacIv-fos time course experiments.

\section{Discussion}

The process of cellular transformation involves complex alterations of gene expression regulation. This level of complexity raises the challenge of identifying the gene expression changes that are most relevant to mechanisms of tumorigenesis. The challenge is especially evident in studies of primary tumor specimens in which the analyzed material represents the end state of a progressive series of events rather than a snapshot of the process itself. Cell-based model systems of transformation and tumorigenesis offer an opportunity to experimentally simulate steps along the pathway of transformation; however, these systems are subject to their own set of caveats including gene expression changes resulting from variation inherent to cell culture conditions and clonal variation. In the study described here, we attempted to provide a more focused view of relevant gene expression changes associated with oncogenic cellular transformation by analyzing a conditional cell-based model system 
A.
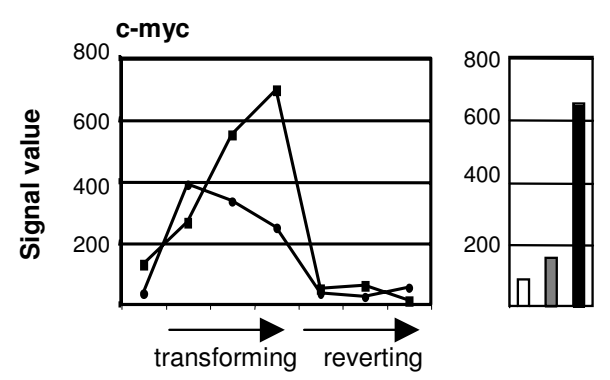

Ral A

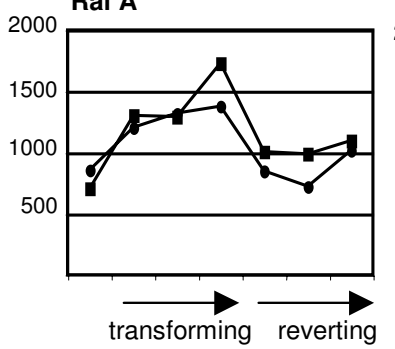

MMP10

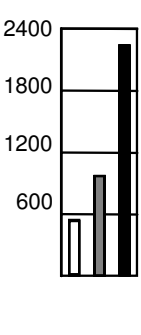

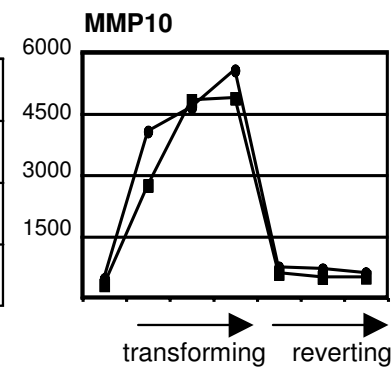

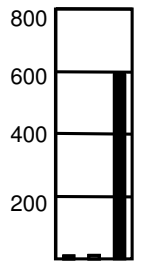

B.
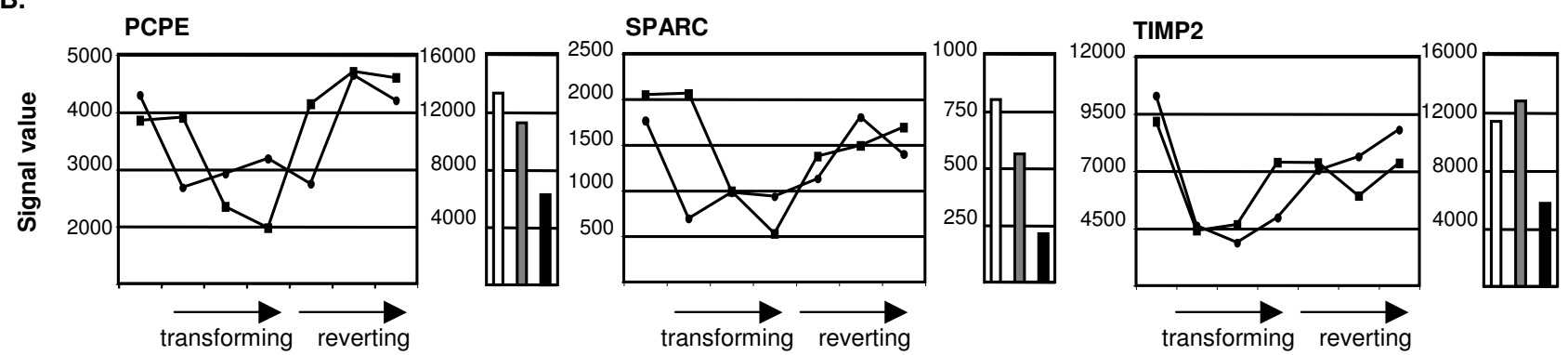

Figure 4

Expression of representative genes conditionally regulated in Laclv-fos cells and stably regulated in FBJ/R cells, but not CMVc-fos cells. Expression patterns of selected genes upregulated (A) or downregulated (B) specifically during conditional transformation, as well as in cells stably transformed by v-Fos. Gene names are indicated above each pair of graphs. Line graphs indicate raw signal values obtained from the two independent Laclv-fos time course experiments. Time points at 24, 48 and 72 hours in the absence of IPTG (transforming) and at 24, 48 and 72 hours after the re-addition of IPTG (reverting) are indicated by arrows below each line graph. Bar graphs indicate raw signal values obtained in microarray analyses of the parental 208F fibroblast cell line (white), the stably transformed c-Fos cell line (CMVc-fos; grey) and the stably transformed vFos cell line (FBJ/R; black).

in which morphological transformation and reversion is experimentally manipulated.

Sustained expression of fos results in the differential expression of a large number of genes $[7,9,11]$. By analyzing gene expression at time points throughout the conditional cellular transformation and reversion of LacIv-fos cells and by arranging these patterns into SOMs, we have clustered differentially expressed genes into cohorts with common temporal patterns of expression. This type of clustering allows identification of groups of genes with expression patterns that are consistent with a potential functional role in the process of morphological transformation. For example, the exogenous v-fos transcript itself is clustered within the conditionally upregulated group 8 . This group also includes the previously reported Fos-regulated target genes such as ezrin [19] and Fra-1 $[20,21]$ genes shown to be strongly upregulated in response to fos-induced transformation $[19,21]$. Interestingly, independent genes with functionally related roles in cellular transformation fall into different expression pattern bins. For example, ezrin and CD44 form a complex that plays an active role in aspects of tumor progression and metastasis such as tumor-endothelium interactions, 
cell migration and cell adhesion [22] but the expression patterns of these two genes differ somewhat. The expression of CD44 is more transiently induced (group 7); this characteristic is consistent with features of a gene whose expression is induced in response to serum stimulation. In contrast, ezrin expression is maintained at its maximal level throughout the 3-day morphological transformation period and rapidly returns to its initial expression levels upon repression of exogenous v-fos expression (group 8). These results demonstrate that coordinated yet distinct programs of gene expression regulation can be initiated to affect the expression of genes that function in common mechanisms of cellular transformation.

Our analysis has revealed additional conditionally regulated genes that have not been previously associated directly with Fos or AP-1. For example, PRL-1 is a protein tyrosine phosphatase originally identified as an immediate-early gene in liver regeneration [23]. PRL-1 expression is elevated in cells stably transformed by either c-fos or vfos, and it is conditionally upregulated during LacIv-fos transformation (group 8). PRL-1 promotes cell migration and invasion in vitro and its expression is elevated in a number of cancer cell lines. PRL-1 is also involved in regulation of progression through mitosis, possibly by modulating spindle dynamics $[24,25]$.

Sustained expression of c-Fos is capable of initiating transformation [7]; however, in our study a comparison of cFos transformed cells (CMVc-fos) with v-Fos transformed cells revealed additional differential gene regulation events in cells transformed by v-Fos relative to cells transformed by c-Fos. For example, expression signal values of both c-myc and the Ras GTPase, RalA, were slightly greater in CMVc-fos cells relative to 208F cells. However, expression of these genes was dramatically upregulated ( $>2$ fold) in FBJ/R cells. Likewise, PCPE and SPARC expression was slightly downregulated in CMVc-fos cells, but were dramatically repressed in FBJ/R cells. In contrast, two genes involved in enzymatic regulation of the extracellular matrix were differentially expressed only in FBJ/R cells. Expression of the matrix metalloproteinase MMP10 and the metalloproteinase inhibitor TIMP2 was specifically upregulated and downregulated in v-fos-transformed cells, respectively. Interestingly, MMP10 was previously identified as a gene whose expression was upregulated gene in cells stably transformed by FBR-v-fos [14]. The mechanisms responsible for differential expression of these genes specifically in v-fos-transformed cells are currently unknown. Some of these genes may be direct targets of v-Fos, but not of c-Fos, or targets of secondary transcription regulatory factors whose expression is deregulated specifically in v-fos-transformed cells. Alternatively, the expression of some genes may be affected by both c-Fos and v-Fos, but to a higher degree in v-fos-transformed cells.

The identification of 28 conditionally downregulated probe sets specific to v-Fos transformation raises the possibility that loss of expression of a subset of these genes may be due to chromatin modifying mechanisms leading to gene silencing. Included in our analysis are several genes previously implicated as targets for gene silencing via epigenetic repression. For example, the extracellular matrix molecule, SPARC, is downregulated in c-jun-transformed primary rat embryo fibroblasts [26]. Also, in v-juntransformed chick embryo fibroblasts, reduction in SPARC mRNA levels has been shown to be due in part to the formation of a DNA-Sp1/3-v-Jun chromatin-associated complex [27]. Tissue inhibitor of metalloprotease 2 (TIMP-2), an endogenous inhibitor of MMP-2, has been shown to inhibit invasion and metastasis [28] and overexpression of TIMP-2 inhibited growth and reduced invasive potential in tumor cells [29]. TIMP-2 is subject to aberrant promoter hypermethylation in human cervical cancer cells and increased methylation favors development of primary cervical cancers [30]. Interestingly, treatment of human neuroblastoma cells with the DNA methyltransferase inhibitor 5-azacytidine (5-AzaC) restored TIMP-2 expression and resulted in a reduction of in vitro invasiveness [31]. The precise role of $v$-fos in mediating gene silencing in the conditional system is not clear; however, our system represents a tool for identifying candidate genes that are subject to epigenetic modification during the process of oncogenesis, as well as a conditional cellular system that can be employed to investigate the temporal mechanisms underlying these regulatory events.

\section{Conclusion}

Technological advances in the ability to analyze gene expression profiles on an increasingly global scale have contributed significantly to a more comprehensive view of the complex transcriptional networks that go awry in tumor cells [32,33]. Studies of in vitro cellular model systems of oncogenic transformation have provided a wealth of information relevant to both normal signal transduction pathways and tumorigenic mechanisms. However, the large number of genes differentially expressed in these model systems often complicates the identification of the most promising candidate genes for further study. Our web-accessible database of transcriptional changes detected in the conditional v-fos system provides a powerful tool to identify cohorts of gene candidates associated with specific cellular events during the process of transformation and reversion. 


\section{Methods \\ Cell culture and $v$-fos transformation and reversion time course}

All cells were maintained in DMEM supplemented with $10 \%$ fetal calf serum, L-glutamine and penicillin/streptomycin at $37^{\circ} \mathrm{C}$ in the presence of $5 \% \mathrm{CO}_{2}$. Establishment of the stably transformed cell lines, FBJ/R and CMVc-fos, was described previously [9]. Establishment of the LacIvfos conditional cell line and the conditions required for the transformation/reversion time course were described previously [11]. Briefly, for the v-fos transformation and reversion time course experiment, total RNA was extracted from LacIv-fos cells cultured in the presence of $5 \mathrm{mM}$ IPTG at time zero; 24, 48 and 72 hours after removal of IPTG (transforming); and 24, 48 and 72 hours after the re-addition of $5 \mathrm{mM}$ IPTG (reverting). Total RNA was extracted from cells by using Tripure reagent according to the manufacturer's instructions (Roche). The integrity of all RNA samples was verified by using an Agilent 2100 Bioanalyzer.

\section{Western blot and immunofluorescence analyses}

Expression of Fos protein during transformation and reversion was evaluated by Western blotting analysis using an antibody directed against Fos [7]. Whole-cell lysates were prepared in lysis buffer $(20 \mathrm{mM}$ Tris $\mathrm{pH} 7.5$, $100 \mathrm{mM} \mathrm{NaCl}, 0.5 \% \mathrm{NP}-40$, protease inhibitor cocktail [Roche]) and separated on a 12\% SDS-polyacrylamide gel. For immunofluorescence microscopy, cells were fixed in $4 \%(\mathrm{w} / \mathrm{v})$ formaldehyde. Fixed cells were incubated with primary mouse monoclonal anti-vinculin antibody (Sigma; dilution of 1:200) and phalloidin-congjugated to Alexa-568 (Molecular Probes). An anti-mouse secondary antibody (dilution of 1:1000) conjugated to Alexa-488 (Molecular Probes) was used to detect vinculin.

\section{Microarray analyses}

RNA samples were processed for hybridization without amplification and hybridized to Affymetrix Rat Genome U34A GeneChips that include probe sets representing approximately 7,000 annotated genes and 1,000 EST clones [34]. Labelling and hybridization were preformed as described [35]. GeneChips were scanned using a laser confocal scanner (Agilent Technologies) and images were analyzed using Affymetrix Microarray Suite v.5.0. Datasets were standardized by global scaling of the average fluorescent intensities of all probe sets to a constant target value of 500 for all arrays. Quality control parameters for each hybridization were within MIAME compliant specifications [36]. A variance filter was applied to the dataset to remove data from probe sets representing genes that were not expressed throughout the time course and to standardize expression values for genes whose expression was scored absent at particular time points [37]. Data that were derived from probe sets that reported absent change calls at all seven time points were removed from the analysis. To standardize expression values of genes whose expression was scored as absent at fewer than seven time points, we converted the signal values that corresponded to absent change calls to a value of 1 . Genes scored as differentially expressed in CMVc-fos cells and FBJ/R cells relative to $208 \mathrm{~F}$ cells had a signal $\log$ ratio $\geq 1$ (increased) or $\leq-1$ (decreased), and a change p-value $<0.001$ (increased) or $>0.99$ (decreased). For visualization of specific profiles of gene expression during the LacIv-fos conditional transformation and reversion time course, signal values obtained at each LacIv-fos time point were plotted by using Microsoft Excel.

\section{SOM analysis}

Microarray data that were obtained from the LacIv-fos time course experiments and passed the variance filter were grouped into relative expression pattern bins by the self-organizing map (SOM) program in the GeneMaths software package (version 1.5; Applied Maths, Austin, TX). The SOM analysis was performed by using a $6 \times 3$ node format to allow optimal representation of gene expression patterns in a reasonably small number of independent bins.

\section{The SOM searchable database}

The SOM searchable database has been implemented on an Open Source MySQL 4.0.14 relational database management system. The database has a web interface at http:/ /www.stjuderesearch.org/v-fosSOM/. The web application to query and manage the database is driven with a server-side scripting technology JSP. The entries of this database are generated from the tab-delimited output files of the SOM analysis. Data obtained from the SOM analysis can be queried by three approaches. Users can click on a graphical pattern key to view a list of all genes whose expression profiles match the particular self-organization pattern. Each entry in the list contains two parameters associated with the respective gene probe, i.e., the Affymetrix probe set ID number and a description of the gene. The detailed Affymetrix analysis data for a probe set can be obtained by clicking the link of the respective probe set ID number. The corresponding Affymetrix analysis page provides information about the number of oligonucleotide pairs, the signal magnitude, the detection status, and the detection $\mathrm{p}$ value at seven different time points derived from 2 independent experiments. Another search method is to query via the GenBank accession number. The query by accession number is based on the fact that each Affymetrix probe set ID number in our current experiments corresponds to a gene accession number. If an accession number in a query matches with any part within a probe set ID number, the entry containing the probe set ID number, its SOM pattern, and gene description is displayed. Hyperlinks are provided for both probe set ID 
numbers and SOM pattern. By following the link for a probe set ID number, the user can view the detailed Affymetrix analysis data for a selected gene probe; by following the link for an SOM pattern, the user can view the detailed Affymetrix analysis data for all the gene probes within the selected pattern. Also, a keyword-based text search is included in the database. A search for keywords in the paragraph of the gene description field is conducted in a case-insensitive manner. The search-by-keyword method is facilitated by the use of Boolean operations. The search can narrow or broaden quickly the results of the search by combining two or more keyword(s) with only one type of Boolean (and / or) operation.

\section{Authors' contributions}

JMO prepared cells, purified RNA, and analyzed the microarray data, SDF performed immunofluoresence microscopy and Western blot analysis, and HR designed the web-accessible database. JMO, SDF, HR, and TC composed the manuscript.

\section{Additional material}

\section{Additional File 1}

Genes differentially expressed (i.e., expression varied by a factor of at least 2) in $C M V c$-fos cells and in $F B J / R$ cells and conditionally regulated in LacI $v$-fos cells.

\section{Click here for file}

[http://www.biomedcentral.com/content/supplementary/14764598-4-19-S1.doc]

\section{Acknowledgements}

We thank John J. Morris and the Clinical Applications Core Technology Center at St Jude Children's Research Hospital (SJCRH) for generating microarray data and providing advice about data analysis. We also thank Julia Cay Jones of Scientific Editing at SJCRH for editorial comments and The Hartwell Center at SJCRH for advice on web-site preparation and hosting. This work was supported by the National Institute of Health grant ROI CA 84I39 (TC) and the American Lebanese Syrian Associated Charities (ALSAC).

\section{References}

I. Morgan Jl, Curran T: Stimulus-transcription coupling in the nervous system: involvement of the inducible proto-oncogenes fos and jun. Annu Rev Neurosci I991, I 4:421-45I.

2. Curran T: The fos oncogene. In The oncogene handbook Edited by: Reddy EP, Shalka AM, Curran T. Amsterdam: Elsevier; 1988:307-325.

3. Finkel MP, Biskis BO, Jinkins PB: Virus induction of osteosarcomas in mice. Science 1966, I5 I:698-701.

4. Curran T, Peters G, Van Beveren C, Teich NM, Verma IM: FBJ murine osteosarcoma virus: identification and molecular cloning of biologically active proviral DNA. J Virol 1982, 44:674-682

5. Curran T, Teich NM: Candidate product of the FBJ murine osteosarcoma virus oncogene: characterization of a 55,000dalton phosphoprotein. J Virol 1982, 42: | |4- 122.

6. Curran T, Miller AD, Zokas L, Verma IM: Viral and cellular fos proteins: a comparative analysis. Cell 1984, 36:259-268.
7. Miao GG, Curran T: Cell transformation by c-fos requires an extended period of expression and is independent of the cell cycle. Mol Cell Biol 1994, I4:4295-4310.

8. Bestor TH, Verdine GL: DNA methyltransferases. Curr Opin Cell Biol 1994, 6:380-399.

9. Bakin AV, Curran T: Role of DNA 5-methylcytosine transferase in cell transformation by fos. Science 1999, 283:387-390.

10. Cross SH, Bird AP: CpG islands and genes. Curr Opin Genet Dev 1995, 5:309-3|4.

II. Ordway JM, Williams K, Curran T: Transcription repression in oncogenic transformation: common targets of epigenetic repression in cells transformed by Fos, Ras or Dnmtl. Oncogene 2004, 23:3737-3748.

12. Hennigan RF, Hawker KL, Ozanne BW: Fos-transformation activates genes associated with invasion. Oncogene 1994, 9:3591-3600.

13. Ozanne BW, McGarry L, Spence HJ, Johnston I, Winnie J, Meagher L, Stapleton G: Transcriptional regulation of cell invasion: AP-I regulation of a multigenic invasion programme. Eur J Cancer 2000, 36:1640-1648.

14. Johnston IM, Spence HJ, Winnie JN, McGarry L, Vass JK, Meagher L, Stapleton G, Ozanne BW: Regulation of a multigenic invasion programme by the transcription factor, AP-I: re-expression of a down-regulated gene, TSC-36, inhibits invasion. Oncogene 2000, 19:5348-5358.

15. Kohonen T: The self-organizing map. Proceedings of the IEEE 1990, 9: |464-|480.

16. Tamayo P, Slonim D, Mesirov J, Zhu Q, Kitareewan S, Dmitrovsky E, Lander ES, Golub TR: Interpreting patterns of gene expression with self-organizing maps: methods and application to hematopoietic differentiation. Proc Natl Acad Sci U S A 1999, 96:2907-29I2.

17. Toronen P, Kolehmainen M, Wong G, Castren E: Analysis of gene expression data using self-organizing maps. FEBS Lett 1999, 45 I : | $42-\mid 46$.

I8. Curran T, Verma IM: FBR Murine osteosarcoma virus. I. Molecular analysis and characterization of a 75,000-dalton gag-fos fusion product. Virology 1984, I35:2 18-228.

19. Lamb RF, Ozanne BW, Roy C, McGarry L, Stipp C, Mangeat P, Jay DG: Essential functions of ezrin in maintenance of cell shape and lamellipodial extension in normal and transformed fibroblasts. Curr Biol 1997, 7:682-688.

20. Cohen DR, Curran T: fra-I: a serum-inducible, cellular immediate-early gene that encodes a fos-related antigen. Mol Cell Biol 1988, 8:2063-2069.

21. Braselmann S, Bergers G, Wrighton C, Graninger P, Superti-Furga G, Busslinger M: Identification of Fos target genes by the use of selective induction systems. J Cell Sci Suppl 1992, 16:97-109.

22. Martin TA, Harrison G, Mansel RE, Jiang WG: The role of the CD44/ezrin complex in cancer metastasis. Crit Rev Oncol Hematol 2003, 46: 165-186.

23. Diamond RH, Cressman DE, Laz TM, Abrams CS, Taub R: PRL-I, a unique nuclear protein tyrosine phosphatase, affects cell growth. Mol Cell Biol 1994, I4:3752-3762.

24. Zeng Q, Dong JM, Guo K, Li J, Tan HX, Koh V, Pallen CJ, Manser E, Hong W: PRL-3 and PRL-I promote cell migration, invasion, and metastasis. Cancer Res 2003, 63:2716-2722.

25. Wang J, Kirby CE, Herbst R: The tyrosine phosphatase PRL-I localizes to the endoplasmic reticulum and the mitotic spindle and is required for normal mitosis. J Biol Chem 2002, 277:46659-46668.

26. Mettouchi A, Cabon F, Montreau N, Vernier P, Mercier G, Blangy D, Tricoire $H$, Vigier $P$, Binetruy $B$ : SPARC and thrombospondin genes are repressed by the c-jun oncogene in rat embryo fibroblasts. EMBO J 1994, 13:5668-5678.

27. Chamboredon S, Briggs J, Vial E, Hurault J, Galvagni F, Oliviero S, Bos T, Castellazzi M: v-Jun downregulates the SPARC target gene by binding to the proximal promoter indirectly through Sp I/ 3. Oncogene 2003, 22:4047-406I.

28. Montgomery AM, Mueller BM, Reisfeld RA, Taylor SM, DeClerck YA: Effect of tissue inhibitor of the matrix metalloproteinases-2 expression on the growth and spontaneous metastasis of a human melanoma cell line. Cancer Res 1994, 54:5467-5473.

29. Imren S, Kohn DB, Shimada H, Blavier L, DeClerck YA: Overexpression of tissue inhibitor of metalloproteinases-2 retroviral- 
mediated gene transfer in vivo inhibits tumor growth and invasion. Cancer Res 1996, 56:2891-2895.

30. Ivanova T, Vinokurova S, Petrenko A, Eshilev E, Solovyova N, Kisseljov $\mathrm{F}$, Kisseljova $\mathrm{N}$ : Frequent hypermethylation of 5' flanking region of TIMP-2 gene in cervical cancer. Int J Cancer 2004, 108:882-886.

31. Cappabianca L, Farina AR, Tacconelli A, Mantovani R, Gulino A, Mackay AR: Reconstitution of TIMP-2 expression in SH-SY5Y neuroblastoma cells by $\mathbf{5}$-azacytidine is mediated transcriptionally by NF-Y through an inverted CCAAT site. Exp Cell Res 2003, 286:209-218.

32. Harkin DP: Uncovering functionally relevant signalling pathways using microarray-based expression profiling. Oncologist 2000, 5:50I-507.

33. Vogelstein B, Kinzler KW: Cancer genes and the pathways they control. Nat Med 2004, 10:789-799.

34. Affymetrix Website: [http://www.affymetrix.com/products/
(it arrays/specific/rgu34.affx]

35. Affymetrix Website [http://www.affymetrix.com/support/techni $\mathrm{cal} /$ manual/expression manual.affx]

36. Brazma A, Hingamp $P$, Quackenbush J, Sherlock G, Spellman $P$, Stoeckert C, Aach J, Ansorge W, Ball CA, Causton HC, Gaasterland T, Glenisson P, Holstege FC, Kim IF, Markowitz V, Matese JC, Parkinson H, Robinson A, Sarkans U, Schulze-Kremer S, Stewart J, Taylor R, Vilo J, Vingron M: Minimum information about a microarray experiment (MIAME)-toward standards for microarray data. Nat Genet 200I, 29:365-37I.

37. Ross ME, Mahfouz R, Onciu M, Liu HC, Zhou X, Song G, Shurtleff SA, Pounds S, Cheng C, Ma J, Ribeiro RC, Rubnitz JE, Girtman K, Williams WK, Raimondi SC, Liang DC, Shih LY, Pui CH, Downing JR: Gene expression profiling of pediatric acute myelogenous leukemia. Blood 2004, I04:3679-3687.

Publish with Bio Med Central and every scientist can read your work free of charge

"BioMed Central will be the most significant development for disseminating the results of biomedical research in our lifetime. "

Sir Paul Nurse, Cancer Research UK

Your research papers will be:

- available free of charge to the entire biomedical community

- peer reviewed and published immediately upon acceptance

- cited in PubMed and archived on PubMed Central

- yours - you keep the copyright

Submit your manuscript here:

http://www.biomedcentral.com/info/publishing_adv.asp
BiolMedcentral 